ARTí́CULO

\title{
Capacidad de biofiltración de nutrientes y crecimiento de macroalgas utilizando efluentes generados en el cultivo del pez dorado Seriola lalandi (Perciformes: Carangidae)
}

Nutrients biofiltration capacity and growth of macroalgae cultivated in effluents generated in the production of the yellowtail amberjack Seriola lalandi (Perciformes: Carangidae)

\section{Roberto Ramos $^{1 *}$ y Sebastián Gallardo ${ }^{1}$}

${ }^{1}$ Departamento de Ciencias Acuáticas y Ambientales, Facultad de Ciencias del Mar y de Recursos Biológicos, Universidad de Antofagasta, Av. Angamos 601, Antofagasta, Chile

*Autor corresponsal: roberto.ramos@uantof.cl

\begin{abstract}
The removal capacity of dissolved nutrients and growth of the red macroalgae Agarophyton chilense and Mazzaella canaliculata and the green Ulva lactuca were evaluated using the effluents from the Seriola lalandi culture. The three macroalgae species reached high nitrite and phosphate removal values between 84 and $87.5 \%$, respectively. On the other hand, in the removal of ammonia the best results were achieved by $U$. lactuca and $A$. chilense, both with $46.4 \%$. In nitrate removal, $A$. chilense and $M$. canaliculata achieve efficiencies of 40 and $50 \%$, respectively. In relation to the growth achieved by algae, they show that the daily growth rate of $M$. canaliculata registered a loss of biomass quantified at $0.29 \%$, while $U$. lactuca showed an increase of $0.78 \%$ and A. chilense reached the best performance with $2.8 \%$. Based on the results obtained, it is possible to conclude on the feasibility of using macroalgae in the removal of nutrients, especially $A$. chilense, which, also, from the perspective of increasing biomass, the cultivation of $A$. chilense opens expectations to obtain secondary productions from Seriola lalandi fish farming in an integrated production system.
\end{abstract}

Key words: Biofiltration, growth, macroalgae, fish farming effluent

Resumen.- Se evaluó la capacidad de biofiltración de nutrientes y el crecimiento de las especies de macroalgas rodófitas Agarophyton chilense y Mazzaella canaliculata y la clorófita Ulva lactuca utilizando los efluentes del cultivo de Seriola lalandi. Las tres macroalgas utilizadas alcanzan altos valores de remoción de nitrito y fosfato, entre 84,0 y 87,5\%, respectivamente. En cambio, en la biofiltración de amonio los mejores resultados fueron alcanzados por $U$. lactuca y $A$. chilense, ambas con 46,4\%. En la remoción de nitrato, $A$. chilense y $M$. canaliculata, alcanzaron eficiencias del 40 y $50 \%$, respectivamente. En relación al crecimiento alcanzado por las algas, los resultados muestran que la tasa de crecimiento diario de $M$. canaliculata registró una pérdida de biomasa cuantificada en 0,29\%, por su parte $U$. lactuca mostró un incremento de $0,78 \%$ y $A$. chilense alcanzó el mejor rendimiento con $2,8 \%$. Este estudio concluye con la confirmación de la utilización de macroalgas en la remoción de nutrientes a partir de efluentes de piscicultura. Además, desde la perspectiva del incremento de biomasa, el cultivo de $A$. chilense promueve expectativas para la obtención de producciones secundarias a la piscicultura de Seriola lalandi en un sistema integrado de producción.

Palabras clave: Biofiltración de nutrientes, crecimiento, macroalgas, efluente piscicultura

\section{INTRODUCCIÓN}

La aplicación de tecnologías avanzadas para optimizar la producción de la acuicultura intensiva en Chile, han permitido alcanzar un importante desarrollo de la industria, incluyendo beneficios económicos y sociales, aunque no exento de críticas por el impacto ambiental que genera en los lugares donde se practica (SUBPESCA 2003, Fuentes 2014).
La acuicultura como cualquier otra actividad productiva no deja de generar impactos en el ambiente (Vinatea 1999). Dentro de las actividades acuícolas que generan mayor impacto se encuentran los cultivos en estanques, principalmente de peces y camarones, debido a las características de los efluentes generados (PaniaguaMichel \& García 2003). A este respecto, son múltiples los contaminantes contenidos en los efluentes, entre los cuales se describen los componentes disueltos, las heces y restos de alimentos o medicamentos, entre otros (Pardo et al. 2006). 
En muchos sistemas de producción de peces en estanques, solamente el $30 \%$ de los nutrientes suministrados son convertidos en biomasa, y el 70\% restante es acumulado en los sedimentos o es liberado en el efluente (Gross et al. 2000), lo que resulta en contaminación de los ambientes naturales (Neori et al. 1996).

Según Pardo et al. (2006) existen dos formas de minimizar el impacto de los efluentes de la acuicultura en el ambiente, por una parte, reducir a cero las emisiones, implementando sistemas de recirculación de agua y la otra, tratar los efluentes que se generan en el proceso productivo antes de ser vertidos en los ambientes naturales, asegurando una calidad acorde con las exigencias ambientales. A este respecto, desde bastante tiempo se ha trabajado en Chile (Buschmann et al. 1996, 2001, 2005; Soto \& Mena 1999) y a nivel internacional en la búsqueda de tecnologías amigables con el ambiente para tratar los efluentes (Vinatea 1999, Troell et al. 2003, Ramos et al. 2008, 2009). Dentro de estas tecnologías destaca la utilización de estanques de sedimentación (Porrello et al. 2003, Jackson et al. 2003), filtración por moluscos (Jones et al. 2001, 2002; Mugg \& Rice 2003) y la aplicación de procesos de biofiltración por micro y macroalgas (Shpigel \& Neori 1996, Neori et al. 1996, 2000, 2004; Troell et al. 1999, Chopin et al. 2001, Paniagua-Michel \& García 2003, Hernández et al. 2006, Ramos \& Pizarro 2018) entre otras posibilidades.

La utilización de procesos de biofiltración para tratar los efluentes de la acuicultura mediante el empleo de macroalgas, se ha incrementado en los últimos años, considerando la capacidad de estas para asimilar nutrientes. El nitrógeno y el fósforo son considerados los elementos más importantes en relación al crecimiento algal en la mayoría de los ambientes naturales (Pardo et al. 2006), constituyendo a su vez la fracción disuelta principal en los efluentes generados de la piscicultura.

La integración de las algas con peces y/o camarones en la acuicultura, es una alternativa viable para la reutilización de los nutrientes disueltos en los efluentes y para el crecimiento de la biomasa de algas (Chopin et al. 2001). Estas actividades se agrupan bajo el concepto de Acuicultura Multitrófica Integrada (AMTI). Esta aproximación pasa por la valorización de los residuos generados no como un producto final, sino como un elemento más en la integración de factores y componentes biológicos, físicos y químicos que permitan la diversificación de la producción de una forma sostenible (Chopin et al. 2008). Al respecto, Vega et al. (2020) plantean que muchas macroalgas son una importante fuente de compuestos naturales bioactivos, especialmente aquellos que exhiben actividad antioxidante. Los compuestos identificados con actividad antioxidante son principalmente la clorofila y sus derivados, carotenoides, vitamina A y C, fucoxantina, enzimas, aminoácidos similares a las micosporinas, polisacáridos y polifenoles. Estos compuestos se pueden obtener a partir de algas cultivadas a bajo costo en sistemas de Acuicultura Multitrófica Integrada (AMTI). Por su parte, Figueroa et al. (2012) concluyeron que Crassiphycus corneus (antes Hydropuntia cornea) cultivada en efluentes de estanques de peces muestra una alta tasa de biofiltración de nitrógeno inorgánico y acumulación de compuestos nitrogenados comercialmente viables como ficobiliproteínas y aminoácidos similares a las micosporinas.

Hernández et al. (2006) plantean la posibilidad de cultivos de Gracilariopsis longissima a pequeña y gran escala utilizando las aguas provenientes de cultivos intensivos de peces, obteniendo resultados positivos, principalmente con la incorporación de derivados del nitrógeno en su biomasa. Por su parte, Matos et al. (2006) establecieron que Gracilaria bursa-pastoris y Chondrus crispus presentan una elevada capacidad de remoción de nitrógeno. A su vez, Neori et al. (2004), utilizaron la macroalga Ulva lactuca como reguladores de la calidad de agua en sistemas integrados con cultivos de peces, destacando los niveles de crecimiento en las algas, asociados directamente a los altos niveles de nitrógeno. Especies de macroalgas como Ulva lactuca y Gracilaria sensu lato han demostrado su eficacia para reducir la carga de nutrientes en los efluentes y ayudar a mantener la calidad del agua a niveles aceptables (Neori et al. 2004). El género Agarophyton (antes Gracilaria) ha demostrado ser el candidato más atractivo para el cultivo intensivo, debido a su capacidad para alcanzar altos rendimientos y generar producciones secundarias de valor comercial (Chopin et al. 2001, Neori et al. 2004).

Ante la necesidad de desarrollar tecnologías que sean amigables con el ambiente, y que den sustentabilidad al proceso productivo de Seriola con aplicaciones tecnológicas de bajo costo, el objetivo principal del presente estudio fue la evaluación de la capacidad de remoción de nutrientes y crecimiento de tres especies de macroalgas, Agarophyton chilense, Mazzaella canaliculata y Ulva lactuca, utilizando los efluentes generados de la piscicultura de Seriola lalandi.

\section{Materiales Y MÉTODOS}

El experimento se realizó en la Facultad de Ciencias del Mar y de Recursos Biológicos de la Universidad de Antofagasta, Antofagasta, Chile. Se evaluó el proceso de biofiltración de nutrientes y el crecimiento algal en un periodo experimental de 7 días. El tiempo experimental fue establecido con el propósito de evitar el deterioro de la biomasa algal en los reactores biológicos, debido a la alta radiación solar propia del norte de Chile, además de evitar el agotamiento de nutrientes al emplear sistemas estáticos sin renovación de agua. 
Las macroalgas $A$. chilense, M. canaliculata y $U$. lactuca fueron colectadas mediante buceo apnea desde el submareal, a una profundidad promedio de $2 \mathrm{~m}$, en el sector denominado "Bolsico" de la Caleta Errázuriz (2328'22,45'S; 70³6'20,11'O), distante $50 \mathrm{~km}$ al norte de la ciudad de Antofagasta. En el laboratorio, las macroalgas fueron mantenidas por $24 \mathrm{~h}$ en un estanque de $2.500 \mathrm{~L}$ con aireación constante y flujo abierto de agua de mar. Antes del inicio experimental, se seleccionaron aquellas frondas de cada especie de alga que presentaban las mejores características externas de coloración, tamaño y cantidad de epífitos presentes.

El efluente utilizado en el experimento fue obtenido a partir de estanques de cultivo del tipo australiano con volumen total de $180 \mathrm{~m}^{3}$ que mantenían 15 ejemplares reproductores de Seriola lalandi con un peso promedio de $9 \mathrm{~kg}$, localizados en el área de cultivos del Departamento de Ciencias Acuáticas y Ambientales, Universidad de Antofagasta. Para colectar el efluente fueron simulados cambios de agua, dejando salir agua de fondo que se almacenó en un estanque de $2.500 \mathrm{~L}$ para luego ser homogenizada con agitación mecánica y bombeada a los estanques de experimentación.

En el proceso de remoción de nutrientes y crecimiento, se emplearon 9 estanques experimentales y 3 estanques control, todos ellos de acrílico transparente con formato cilindro cónico y un volumen total de 50 L. Cada especie algal fue sembrada en los estanques en triplicado con 500 $\mathrm{g}$ de biomasa, correspondiente a una densidad de $10 \mathrm{gL}^{-1}$ peso húmedo. Para contrastar los resultados de remoción de nutrientes, fueron utilizados 3 estanques control que contenían solamente efluente, sin algas. El diseño experimental utilizado en la remoción de nutrientes y crecimiento algal se muestran en la Figura 1.

Todos los estanques experimentales estaban provistos de aireación constante para mantener las algas y nutrientes en suspensión.

Terminado el proceso de remoción y crecimiento, en el séptimo día experimental, se tomaron muestras de agua de $500 \mathrm{~mL}$ en duplicado, desde cada unidad experimental y estanques control para realizar los análisis de nutrientes. Para evaluar la eficiencia de remoción (ER) de nutrientes, expresados en porcentaje, se contrastaron las concentraciones de nutrientes de las unidades experimentales y los estanques control, utilizando la relación propuesta por PaniaguaMichel \& García (2003):

$$
E R(\%)=\left[\frac{(C N C-C N E)}{C N C}\right] 100
$$

donde, $\mathrm{CNC}=$ Concentración nutriente estanque control y $\mathrm{CNE}=$ Concentración nutriente estanque experimental.

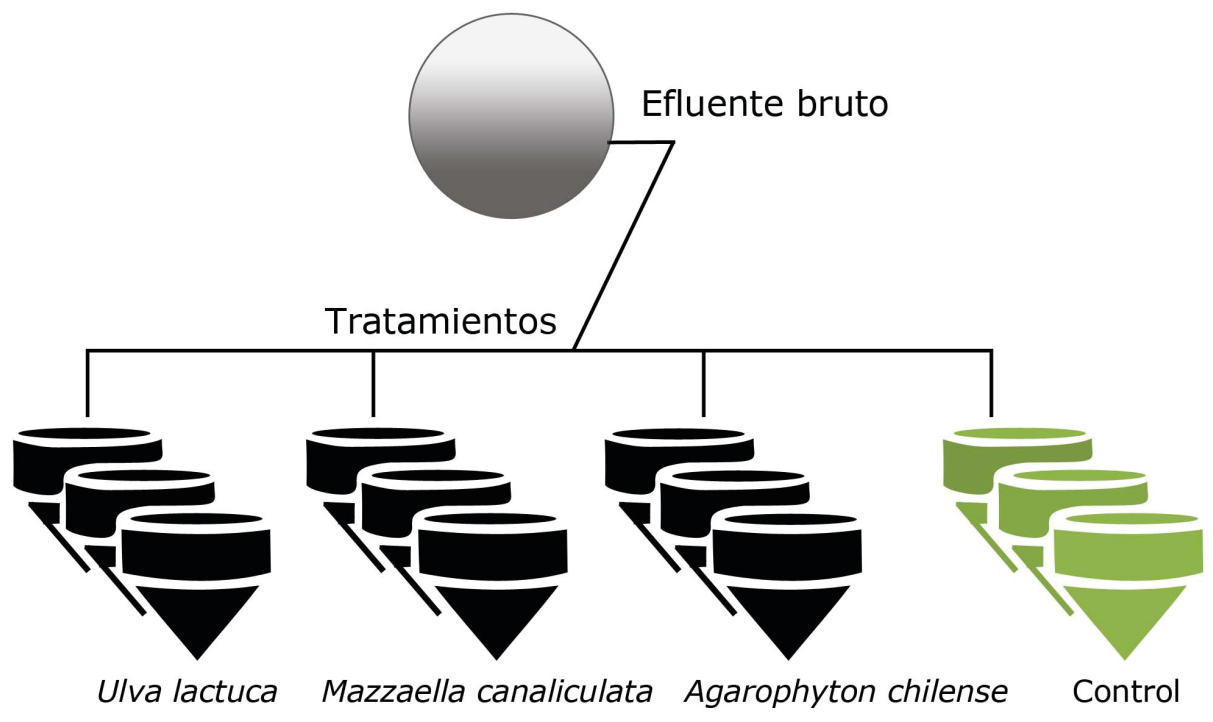

Figura 1. Diseño experimental para los tres tratamientos con las macroalgas U. lactuca, M. canaliculata, $A$. chilense y estanque control / Experimental design for the three treatments with the macroalgae $U$. lactuca, $M$. canaliculata, $A$. chilense and control pond 
Diariamente en cada estanque experimental y control, fueron monitoreadas la temperatura, salinidad, $\mathrm{pH}$ y oxígeno disuelto con un equipo multiparámetro $\mathrm{HACH}$ modelo HI 9828. En cuanto a la intensidad de luz, esta fue medida en la parte superior de cada unidad experimental en tres (3) horarios, 11:00, 15:00 y 19:00 h, utilizando un luxómetro marca HANNA® HI 97500. Los nutrientes disueltos, amonio, nitrito, nitrato y fosfato fueron determinados utilizando un fotocolorímetro Espectroquant Nova 60 (Merck®).

La determinación del porcentaje de crecimiento diario (PCD) de las algas, fue evaluada al final del experimento, estableciendo el incremento de biomasa como factor de crecimiento de acuerdo a la fórmula de Deboer \& Ryther (1977):

$$
P C D(\%)=\left[\frac{(W f-W o) / T)}{W o}\right] 100
$$

donde, $W f$ es el peso húmedo final, Wo es el peso húmedo inicial, $T$ el tiempo en días.

Los resultados obtenidos fueron analizados empleando análisis de varianza (ANDEVA) de una vía para determinar diferencias estadísticas entre los tratamientos. Previo a la aplicación del ANDEVA los datos de porcentajes de remoción de nutrientes fueron transformados a sus equivalentes arco seno, además se determinó la normalidad de los datos y homogeneidad de la varianza mediante la aplicación del test de Bartlet y Kolmogorov-Smirnov, respectivamente. Cuando fueron determinadas diferencias significativas en el análisis de varianza fue aplicado el test de Tukey $(P<0,05)$. En todos los análisis estadísticos se utilizó el software Assistat versión 7.5 beta (Silva \& Azevedo 2006)

\section{Resultados}

Los parámetros físico-químicos del agua en los distintos tratamientos se mantuvieron estables. La temperatura registró valores que oscilaron entre 25,5 y $25,6{ }^{\circ} \mathrm{C}$. En cuanto al $\mathrm{pH}$ este parámetro registró valores entre 7,9 y 8,0 , la salinidad osciló entre 34,7 y 35,3 y finalmente el oxígeno disuelto presentó variaciones entre 4,5 y 4,8 mg $\mathrm{O}_{2} \mathrm{~L}^{-1}$, como se observa en la Tabla 1.
Tabla 1. Valores promedios de salinidad, temperatura $\left({ }^{\circ} \mathrm{C}\right), \mathrm{pH}$ y oxígeno disuelto (OD) para todos los tratamientos durante la etapa experimental / Mean values of the salinity, temperatura $\left({ }^{\circ} \mathrm{C}\right), \mathrm{pH}$ and dissolved oxygen (OD) for all treatments during of the experimental stage

\begin{tabular}{lcccc}
\hline $\begin{array}{c}\text { Especies de } \\
\text { macroalgas }\end{array}$ & Salinidad & $\begin{array}{c}\text { Temperatura } \\
\left({ }^{\circ} \mathrm{C}\right)\end{array}$ & $\mathrm{pH}$ & $\begin{array}{c}\mathrm{OD} \\
\left(\mathrm{mg} \mathrm{O}_{2} \mathrm{~L}^{-1}\right)\end{array}$ \\
\hline U. lactuca & $34,7 \pm 0,028$ & $25,6 \pm 0,091$ & $7,9 \pm 0,012$ & $4,7 \pm 0,160$ \\
A. chilense & $35,0 \pm 0,148$ & $25,5 \pm 0,082$ & $8,0 \pm 0,066$ & $4,5 \pm 0,061$ \\
M. canaliculata & $35,3 \pm 0,156$ & $25,5 \pm 0,305$ & $7,9 \pm 0,084$ & $4,8 \pm 0,217$ \\
\hline
\end{tabular}

\section{INTENSIDAD DE LUZ}

Los resultados de la intensidad de luz mostraron que los valores promedios más elevados se presentaron en los horarios de 15:00 y 11:00 h con un máximo de 563,6 $\mu \mathrm{mol}$ $\mathrm{m}^{-2} \mathrm{~s}^{-1}$, y los valores promedios más bajos se verificaron en el horario de las 19:00 h con 123,0 $\mu \mathrm{mol} \mathrm{m}^{-2} \mathrm{~s}^{-1}$, como se presenta en la Figura 2.

\section{REMOCIÓN DE NUTRIENTES}

Los resultados de eficiencia de remoción de nutrientes en las unidades experimentales en relación al estanque control se muestran en la Tabla 2. Los mejores desempeños en la eficiencia de remoción de amonio se alcanzaron con $U$. lactuca y A. chilense, llegando a obtener un valor de 46,4\% para ambas especies de alga. Por su parte, M. canaliculata alcanzó una remoción de $32,1 \%$, presentando diferencia significativa $(P<0,05)$ con los tratamientos anteriores.

En la remoción de nitrito, los tratamientos no presentaron diferencia significativa $(P>0,05)$, alcanzando un valor común de $87,5 \%$ para las tres especies de algas.

En el caso de los nitratos, se observaron diferencias significativas entre los tratamientos $(P<0,05)$. La especie $U$. lactuca registró el menor valor de eficiencia de remoción con $20,0 \%$, mientras que $A$. chilense y $M$. canaliculata alcanzaron 40,0 y 50,0\% respectivamente, no presentando diferencias significativas entre sí.

Respecto de la eficiencia de remoción de fosfato, los tres tratamientos no presentaron diferencia significativa $(P$ $>0,05)$. Los valores registrados fueron bastante similares para los tres tratamientos, es así como $U$. lactuca y $M$. canaliculata alcanzaron $87,3 \%$ y $A$. chilense $84,6 \%$ de eficiencia de remoción. 


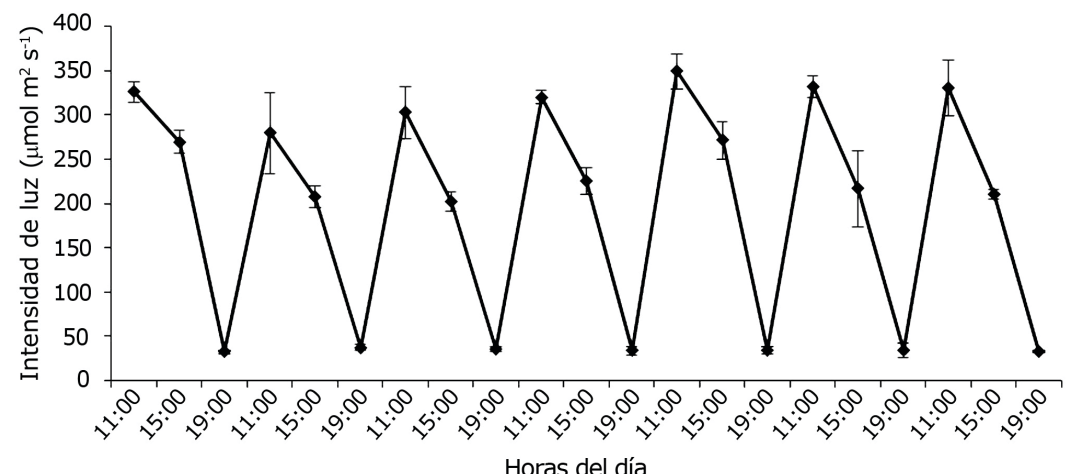

Figura 2. Intensidad de luz, expresados como $\mu \mathrm{mol} \mathrm{m}^{-2} \mathrm{~s}^{-1}$ registrados a lo largo de la etapa experimental / Light intensity, expressed as $\mu \mathrm{mol} \mathrm{m} \mathrm{m}^{-2} \mathrm{~s}^{-1}$ recorded along the experimental condition

Tabla 2. Valores promedios $(n=3 \pm D E$ ) de eficiencia de remoción de nutrientes disueltos durante la etapa experimental / Average values $(n=3 \pm S D$ ) of efficiency of removal of dissolved nutrient during experimental stage

\begin{tabular}{lcccc}
\hline \multicolumn{1}{c}{ Tratamientos } & $\begin{array}{c}\text { Amonio } \\
\left(\mathrm{mg} \mathrm{L}^{-1}\right)\end{array}$ & $\begin{array}{c}\text { Nitrito } \\
\left(\mathrm{mg} \mathrm{L}^{-1}\right)\end{array}$ & $\begin{array}{c}\text { Nitrato } \\
\left(\mathrm{mg} \mathrm{L}^{-1}\right)\end{array}$ & $\begin{array}{c}\text { Fosfato } \\
\left(\mathrm{mg} \mathrm{L}^{-1}\right)\end{array}$ \\
\hline Estanque control & $0,28 \pm 0,04$ & $0,16 \pm 0,02$ & $0,10 \pm 0,02$ & $2,60 \pm 0,14$ \\
Estanque Ulva lactuca & $0,15 \pm 0,04$ & $0,02 \pm 0,01$ & $0,08 \pm 0,03$ & $0,33 \pm 0,06$ \\
Eficiencia de remoción (\%) & $46,4^{\mathrm{a}}$ & $87,5^{\mathrm{a}}$ & $20,06^{\mathrm{b}}$ & $87,3^{\mathrm{a}}$ \\
Estanque Agarophyton chilense & $0,15 \pm 0,02$ & $0,02 \pm 0,01$ & $0,06 \pm 0,02$ & $0,40 \pm 0,00$ \\
Eficiencia de remoción (\%) & $46,4^{\mathrm{a}}$ & $87,5^{\mathrm{a}}$ & $40,0^{\mathrm{a}}$ & $84,6^{\mathrm{a}}$ \\
Estanque Mazzaella canaliculata & $0,19 \pm 0,05$ & $0,02 \pm 0,01$ & $0,05 \pm 0,01$ & $0,33 \pm 0,06$ \\
Eficiencia de remoción (\%) & $32,1^{\mathrm{b}}$ & $87,5^{\mathrm{a}}$ & $50,0^{\mathrm{a}}$ & $87,3^{\mathrm{a}}$ \\
\hline
\end{tabular}

Letras diferentes en la misma columna muestran diferencias significativas $(P<0,05)$

\section{Crecimiento}

En relación al porcentaje de crecimiento diario de las algas después de los 7 días de experimentación, se pudo observar que $U$. lactuca presentó un incremento de biomasa de $0,78 \%$. Por su parte, Mazzaella canaliculata mostró un crecimiento negativo de $0,29 \%$, con algún grado de deterioro de sus frondas, valores que no fueron considerado en el análisis estadístico. Por último, Agarophyton chilense registró un porcentaje de crecimiento diario de $2,8 \%$, siendo la especie que alcanzó el valor más elevado de las tres especies estudiadas, presentando diferencias significativas $(P<0,05)$ (Fig. 3). En relación a los niveles de producción del sistema, los mayores valores promedios en peso húmedo fueron alcanzados por Agarophyton chilense con $0,28 \mathrm{gL}^{-1} \mathrm{~d}^{-1}$, seguido de Ulva lactuca con un valor promedio de producción de $0,078 \mathrm{gL}^{-1} \mathrm{~d}^{-1} \mathrm{y}$ finalmente Mazzaella canaliculata con un valor de pérdida de biomasa húmeda estimada en $-0,29 \mathrm{gL}^{-1} \mathrm{~d}^{-1}$.

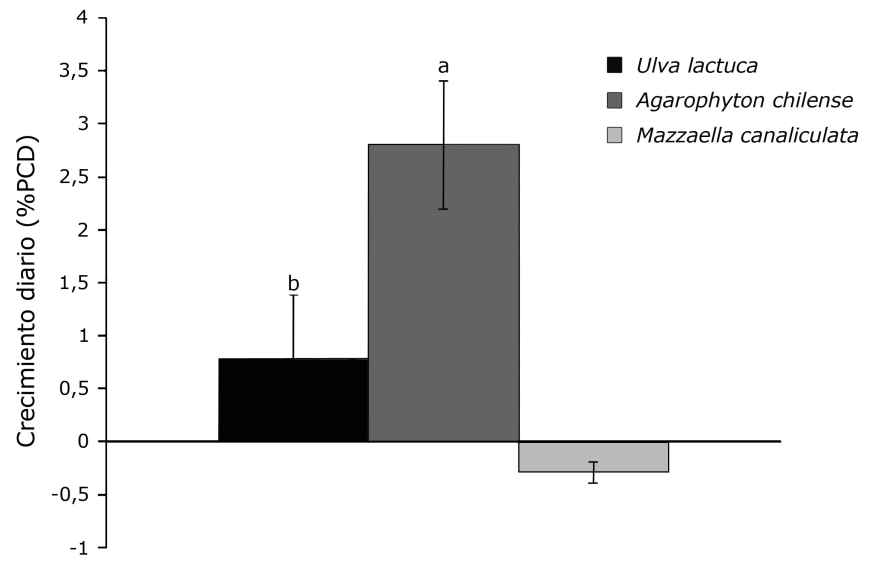

Figura 3. Porcentaje de crecimiento diario (PCD) de tres especies de macroalgas durante la etapa experimental. Letras diferentes muestran diferencias significativas entre tratamientos $(P<0,05) /$ Percentage daily growth (PCD) of three macroalgae species during experimental stage. Different letters show significant differences between treatments $(P<0.05)$ 


\section{Discusión}

Los parámetros físico-químicos del agua no presentaron grandes variaciones durante la etapa experimental, se puede apreciar que, en todos los tratamientos, los niveles de temperatura se mantuvieron estables, no obstante, estos presentaron valores superiores a los del medio natural, atribuible a la alta intensidad de luz que recibieron las unidades experimentales durante el estudio. Al respecto, los valores promedio de intensidad de luz en las condiciones experimentales utilizadas, llegaron a estar sobre los 350 $\mu \mathrm{mol} \mathrm{m} \mathrm{m}^{-2} \mathrm{~s}^{-1}$. Por su parte, los relativamente bajos valores de oxígeno disuelto detectados, estarían asociado al aumento de temperatura del agua en todos los tratamientos, debido a la alta intensidad de luz. En cuanto a la salinidad, esta no presentó grandes diferencias, a pesar de presentar niveles un poco mayores que en el ambiente natural, por efectos de evaporación del agua asociada a los factores anteriores. Por su parte, el pH se mantuvo sin diferencias durante el experimento.

El tratamiento de los efluentes provenientes del cultivo de peces y camarones utilizando macroalgas es considerado efectivo en la reducción de los nutrientes disueltos (Neori et al. 1996, 2000; Troell 1999, Chopin et al. 2001, PaniaguaMichel \& García 2003, Schuenhoff et al. 2003, Troell et al. 2003, Matos et al. 2006, Pardo et al. 2006).

Para el caso de la remoción del amonio, es conocida la preferencia de las algas por este nutriente (Marshall 1991, Lobban \& Harrison 1994, Neori et al. 2004), además, los datos sugieren que la máxima tasa de absorción se logra en los efluentes donde las concentraciones de este nutriente son elevadas (Hernández et al. 2006). Estudios realizados por Shpigel \& Neori (1996) argumentan que la utilización de los géneros Ulva y Agarophyton en sistemas integrados con otros organismos es efectivo en la reducción del amonio. Según Neori et al. (1996) plantean que Ulva lactuca sería un buen biofiltro disminuyendo efectivamente los niveles de amonio. Iguales respuestas han sido establecidas para los géneros Mazzaella y Agarophyton por Matos et al. (2006).

En sistemas integrados de cultivo de peces, abalones y algas, Neori et al. (2000) estimó que $U$. lactuca remueve hasta un $80 \%$ el total de amonio presente en el medio. De igual forma, Troell et al. (2003) indicaron que el cultivo integrado de la dorada Sparus aurata con Ulva lactuca, alcanzó una remoción de $85 \%$ del amonio disponible. Por su parte, Schuenhoff et al. (2003) registraron una remoción de $70 \%$ del amonio presente en un cultivo integrado por Ulva lactuca y peces. En el presente estudio, Ulva lactuca alcanzó valores de remoción de amonio de $46,4 \%$, resultados inferiores a los reportados por los autores anteriores, lo cual se explicaría por la baja concentración de amonio contenidas en el efluente utilizados, tal como lo sugiere Hernández et al. (2006). No obstante, el alcanzar casi un 50\% de remoción del amonio presente en el sistema no deja de ser un resultado positivo e interesante desde el punto del beneficio ambiental.

Respecto del género Agarophyton, Jones et al. (2001) establecieron que la remoción de amonio desde efluentes de cultivo de camarones oscilaba entre 2 y $76 \%$. Matos et al. (2006) establecieron que Gracilaria bursa-pastoris es eficiente en la remoción de amonio, llegando a valores de remoción de $76,7 \%$ del total presente en el efluente del cultivo de los peces Scophathalmus maximus y Dicentrarchus labrax. Comparados los resultados de remoción de amonio alcanzados por el tratamiento con A. chilense de $46,4 \%$, se puede concluir que esta menor eficiencia de remoción es atribuible a la baja concentración $\left(0,28 \mathrm{mg} \mathrm{L}^{-1}\right)$ en el efluente, como fue mencionado anteriormente para Ulva lactuca.

Por su parte, Matos et al. (2006) estudiando la capacidad de absorción de amonio del género Mazzaella, establecieron que este tiene menor eficiencia que el género Agarophyton, lo que también fue constatado en este estudio. No obstante, ambas algas no presentaron diferencias significativas en la remoción de amonio $(P$ $>0,05)$, llegando Mazzaella canaliculata a un valor de eficiencia de remoción de 32,1\%, a pesar de observarse algún deterioro en las frondas.

En este estudio, las tres especies de macroalgas no presentaron diferencias significativas $(P>0,05)$ en la remoción de nitritos. Si bien el nitrito es un producto intermedio entre la transformación del amonio en nitrato, según Timmons et al. (2002) la presencia de nitritos en el sistema solamente precederá a la aparición de nitratos. Además, la remoción de nitrito para las tres macroalgas alcanzó el mismo valor con $87,5 \%$ del total presente en el sistema.

En la remoción de nitratos, según Pereira et al. (2006) el alga roja Porphyra dioica en sus estadios iniciales de desarrollo, es altamente efectiva en su capacidad de remoción de nitratos, alcanzando valores de 99,9\% con intensidades de luz de $250 \mu \mathrm{mol} \mathrm{m} \mathrm{m}^{-2} \mathrm{~s}^{-1}$, valores similares a los registrados en nuestros experimentos. La especie $M$. canaliculata registró un valor de remoción de nitrato superior a las otras especies, alcanzando una eficiencia de $50,0 \%$ en la reducción del nutriente, a diferencia de las otras macroalgas que alcanzaron valores de 20,1 y 40,0\% para U. lactuca y A. chilense, respectivamente. Es relevante mencionar que el género Mazzaella tiene mayor preferencia por lo nitratos que por el amonio como fue señalado por Troell et al. (2003). 
Respecto de la remoción de fosfatos, no se observaron diferencias significativas $(P<0,05)$ entre las tres especies, alcanzando elevados valores de remoción, para $U$. lactuca (87,3\%), A. chilense (84,6\%) y M. canaliculata $(87,3 \%)$. Según Hernández et al. (2006), Gracilaria longissima en condiciones de cultivo a pequeña escala, alcanzó una reducción de $62,2 \%$ del fosfato presente en el agua. Por su parte, Porphyra dioica alcanza niveles de remoción de fosfato de $97,7 \%$ en condiciones de baja densidad y con $150 \mu \mathrm{mol} \mathrm{m} \mathrm{m}^{-2} \mathrm{~s}^{-1}$ de intensidad de luz (Pereira et al. 2006).

En cuanto al crecimiento, las algas marinas muestran ciclos estacionarios de crecimiento y reproducción, los cuales se correlacionan con las fluctuaciones estacionales de intensidad de luz, temperatura y la concentración de nutrientes (Marshall 1991). Al respecto, las macroalgas crecen en altas concentraciones de nutrientes, preferentemente de amonio, acumulando internamente altos contenidos de nitrógeno (Marshall 1991, Neori et al. 2004). En las condiciones experimentales del estudio, es importante destacar que el efluente de la piscicultura del pez dorado no presentó niveles elevados de nutrientes disueltos, más bien podrían considerarse bajos si se comparan con los valores encontrados por Ramos et al. (2008) para los efluentes del cultivo del camarón blanco Litopenaeus vannamei, lo cual pudo condicionar los resultados de crecimiento de las tres especies algales evaluadas, debido a la baja disponibilidad de nutrientes disueltos.

En cuanto a los resultados de crecimiento obtenidos por U. lactuca, esta presentó un porcentaje de crecimiento diario de solo 0,78 , lo que puede atribuirse al bajo nivel de nutrientes presentes en el agua residual del cultivo del pez dorado, especialmente nutrientes nitrogenados, elemento indispensable para que las ulvaceas puedan proliferar y generar tejidos. A pesar del bajo nivel de nutrientes presentes en el efluente utilizado, el valor alcanzado es superior a los valores reportados por Van Khoi \& Fotedar (2011) que registraron crecimientos diarios entre 0,28$0,64 \%$ al integrar Ulva lactuca y el camarón Penaeus latisulcatus en un sistema cerrado, durante 42 días. Las algas del género Ulva crecen en diversos ambientes, pero especialmente en zonas con una fuerte contaminación orgánica natural o antrópica (Perez et al. 1992).

En relación al género Agarophyton, se han reportado de crecimiento diario de $3,35 \%$ luego de 10 días de cultivo en estanques, adicionando nutrientes comerciales al medio (Araya \& Salas 1989). Por su parte, Hernández et al. (2006) establecieron valores diarios de $2,4 \pm 0,5 \%$ en condiciones de cultivo utilizando efluentes de piscicultura. En nuestro estudio, se observó un porcentaje de crecimiento diario de 2,8\%, valor comparable a los estudios señalados anteriormente. Para condiciones de cultivo en medio marino, Robledo (1993) reporta un crecimiento diario de $1,3 \%$. Por su parte, Samocha et al. (2015) reportaron porcentajes de crecimientos diarios de Gracilaria tikvahiae entre 0,75 y $1,48 \%$ al integrar el sistema con camarón Litopenaeus vannamei.

Respecto de $M$. canaliculata, los resultados indicaron una pérdida de biomasa de $0,29 \%$, asociado a un proceso de deterioro de los talos algales. Esto último, es coincidente con temperaturas elevadas en el agua de los estanques experimentales, alta intensidad lumínica y bajos niveles de nutrientes disueltos. Las especies submareales del género Mazzaella y Laminaria que habitan la zona submareal, tienen rangos de saturación de luz entre 30-100 $\mu \mathrm{mol}$ $\mathrm{m}^{-2} \mathrm{~s}^{-1}$. A ese respecto, Mazzaella presenta valores de saturación luz en torno de $94 \mu \mathrm{mol} \mathrm{m}{ }^{-2} \mathrm{~s}^{-1}$, lo que incide directamente en la tasa de crecimiento (Lobban \& Wynne 1981). Analizados los resultados obtenidos de eficiencia de remoción de nutrientes disueltos y crecimiento de $A$. chilense, permiten visualizar la posibilidad de utilizar esta especie en procesos de depuración de aguas residuales a través de la absorción de nutrientes, con la posibilidad de obtener producciones secundarias a la piscicultura del pez dorado. Esta afirmación se sustenta en los resultados reportados por autores como Shpigel \& Neori (1996), Chopin et al. (2001), Troell et al. (2003), Paniagua-Michel \& García (2003) y Ramos et al. (2010), quienes reafirman la factibilidad de la aplicación de estas tecnologías para el tratamiento de los efluentes de la acuicultura, de modo de minimizar el impacto ambiental de las aguas residuales y consecuentemente obtener producciones secundarias al cultivo de peces o camarones. Al respecto, Figueroa et al. (2012) concluyen que la utilización del alga roja $H$. cornea cultivada en efluentes de estanques de peces muestra una alta tasa de biofiltración de nitrógeno inorgánico y acumulación de compuestos nitrogenados como ficobiliproteínas y aminoácidos similares a las micosporinas, además de polisacáridos con propiedades inmunoestimulantes.

Las perspectivas positivas que representan los resultados obtenidos con Agarophyton chilense, tanto en la remoción de nutrientes como en crecimiento, permite avanzar hacia la potencial producción de compuestos bioactivos, como lo plantean Vega et al. (2020). Al respecto, Buschmann et al. (2013) indican que Agarophyton chilense es la especie más importante de macroalga cultivada en el litoral costero, destinándose en parte a la producción del ficocoloide agaragar, el cual se usa como aditivo viscosante en la industria alimenticia, farmacéutica y con fines de investigación en biotecnología. En conclusión, los resultados de este estudio sugieren que $A$. chilense presenta una buena capacidad para la eliminación de nutrientes y puede usarse como biofiltro en la piscicultura de Seriola lalandi. Además, la biomasa producida en los estanques a partir de algas cultivadas a bajo costo, en sistemas de Acuicultura Multitrófica Integrada (AMTI), permitiría obtener subproductos de valor industrial, ampliando así los beneficios al ámbito económico. 


\section{LITERATURA CITADA}

Araya C \& O Salas. 1989. Cultivo de Gracilaria sp. en estanques: efecto de dos niveles de luminosidad, movimiento e ingreso de nutrientes. Seminario de Titulo, Ingeniería en Acuicultura, Facultad de Ciencias del Mar y de Recursos Biológicos, Universidad de Antofagasta, Antofagasta, $66 \mathrm{pp}$.

Buschmann A, M Troell, N Kautsky \& L Kautsky. 1996. Integrated tank cultivation of salmonids and Gracilaria chilensis (Gracilariales, Rhodophyta). Hydrobiologia 326/327: 75-82.

Buschmann A, J Correa, R Westeimeier, M HernándezGonzáles \& R Norambuena. 2001. Red algal farming in Chile: a review. Aquaculture 194: 203-220.

Buschmann A, M Hernández-González, C Astudillo, L de la Fuente, A Gutiérrez \& G Aroca. 2005. Seaweed cultivation, product development and integrated aquaculture studies in Chile. World Aquaculture 36(3): 51-53.

Buschmann A, R. Stead, M Hernández-González, S Pereda, J Paredes \& M. Maldonado. 2013. Un análisis crítico sobre el uso de macroalgas como base para una acuicultura sustentable. Revista Chilena de Historia Natural 86: 251264.

Chopin T, A Buschmann, C Halling, M Troell, N Kautsky, A Neori, G Kraemer, J Zertuche-Gonzalez, C Yarish \& C Neefus. 2001. Integrating seaweeds into marine aquaculture systems: a key toward sustainability. Journal of Phycology 37: 975-986.

Chopin T, S Robinson, M Troell, A Neori, A Buschmann \& J Fang. 2008. Multithropic Integration for sustainable marine aquaculture. In: Jorgensen SE \& BD Fath (eds). Encyclopedia of ecology 32: 463-475. Elsevier, Oxford.

Deboer J \& J Rhyter. 1977. Potential yields from a waste recycling algal mariculture system. In: Krauss $\mathrm{R}$ (ed). The marine plant biomass of the Pacific Northwest Coast, pp. 231-249. Oregon State University Press, Corvallis.

Figueroa FL, N Korbee, R Abdala, CG Jerez, M López-de la Torre, L Güenaga, MA Larrubia \& JL Gómez-Pinchetti. 2012. Biofiltration of fishpond effluents and accumulation of N-compounds (phycobiliproteins and mycosporinelike amino acids) versus $\mathrm{C}$-compounds polysaccharides) in Hydropuntia cornea (Rhodophyta). Marine Pollution Bulletin 64(2): 310-318.

Fuentes J. 2014. Evolución del régimen ambiental de la acuicultura en Chile. Revista de Derecho, Pontificia Universidad Católica de Valparaíso 42: 441-477.

Gross A, C Boyd \& C Wood. 2000. Nitrogen transformations and balance in channel catfish ponds. Aquacultural Engineering 24: 1-14.

Hernández I, A Pérez-Pastor, J Vergara, J MartínezAragón, M Fernández-Engo \& N Pérez-Lloréns. 2006. Studies on the biofiltration capacity of Gracilariopsis longissima from microscale to macroscale. Aquaculture 252: 43-53.

Jackson C, N Preston, M Burford \& P Thompson. 2003. Managing the development of sustainable shrimp farming in Australia: The role of sedimentation ponds in treatment of farm discharge water. Aquaculture 226: 23-34.
Jones A, W Dennison \& N Preston. 2001. Integrated treatment of shrimp effluent by sedimentation, oyster filtration and macroalgal absorption: a laboratory scale study. Aquaculture 193: 155-178.

Jones A, N Preston \& W Dennison. 2002. The efficiency and condition of oyster and macroalgae used as biological filters of shrimp pond effluent. Aquaculture Research 33: 1-19.

Lobban CS \& PJ Harrison. 1994. Seaweed ecology and physiology, 366 pp. Cambridge University Press, London.

Lobban CS \& MJ Winne. 1981. The biology of seaweed, 786 pp. University of California Press, Berkeley

Marshall W. 1991. Biología de las algas. Enfoque fisiológico, 236 pp. Limusa, México D.F.

Matos J, S Costa, A Rodríguez, R Pereira \& I Sousa-Pinto. 2006. Experimental integrated aquaculture of fish and red seaweed in Northern Portugal. Aquaculture 252: 31-42.

Mugg J \& M Rice. 2003. The impacts of aquaculture oysters, Crassostrea virginica (Gmelin, 1791) on water column nitrogen and sedimentation: results of a mesocosm study. Aquaculture 220: 407-422.

Neori A, M Krom, S Ellner, C Boyd, D Popper, R Rabinovitch, P Davison, O Dvir, D Zuber, M Ucko, D Angel \& H Gordón. 1996. Seaweed biofilters as regulators of water quality in integrated fish-seaweed culture units. Aquaculture 141: 183-199.

Neori A, M Shpigel \& D Ben-Ezra. 2000. A sustainable integrated system for culture of fish, seaweed and abalone. Aquaculture 186: 279-291.

Neori A, T Chopin, M Troell, A Buschmann, G Kraemer, C Halling, M Shpigel \& C Yarish. 2004. Integrated aquaculture: rationale, evolution and state of the art emphasizing seaweed biofiltration in modern mariculture. Aquaculture 231: 361-391.

Paniagua-Michel J \& O García. 2003. Ex-situ bioremediation of shrimp culture effluent using constructed microbial mats. Aquacultural Engineering 28: 131-139.

Pardo S, H Suárez \& E Soriano. 2006. Tratamiento de efluentes: una vía para la acuicultura responsable. Revista Medicina Veterinaria y Zootecnia (MVZ), Universidad de Córdova 11: 20-29.

Perez R, R Kaas, F Campello, S Arbault \& O Barbaroux. 1992. La culture des algues marines dans le monde, 613 pp. Institut Français de Recherche pour l'Exploitation de la Mer (IFREMER). Service de la Documentation et des Publications (SDP), Plouzané.

Porrello S, M Lenzi, P Tomassetti, E Persia, M Finoai \& I Mercalati. 2003. Reduction of aquaculture wastewater eutrophication by phyto treatment ponds system: II. Nitrogen and phosphorus content in macroalgae and sediment. Aquaculture 219: 531-544.

Ramos R \& P Pizarro. 2018. Crecimiento y capacidad de biorremediación de Chlorella vulgaris (Trebouxiophycea, Chlorophyta) cultivada en aguas residuales generadas en el cultivo del pez dorado Seriola lalandi (Perciformes: Carangidae). Revista de Biología Marina y Oceanografía 53(1): 75-86. 
Ramos R, L Vinatea \& R da Costa. 2008. Tratamiento de efluentes del cultivo de Litopenaeus vannamei por sedimentación y filtración de la ostra Crassostrea rhizophorae. Latin American Journal of Aquatic Research 36(6): 235-244.

Ramos R, L Vinatea, W Seiffert, E Beltrame, J Santos \& R da Costa. 2009. Treatment of shimp effluent by sedimentation and oyster filtration using Crassostrea gigas and C. rhizophorae. Brazilian Archives of Biology and Technology 52(3): 775-783.

Ramos R, L Vinatea, J Santos \& R Da Costa. 2010. Tratamiento de efluentes del cultivo de Litopenaeus vannamei mediante procesos de sedimentación, filtración y absorción. Latin American Journal of Aquatic Research 38(2): 188-200.

Robledo D. 1993. Cultivo, adaptación morfológica y fisiopatología de macroalgas marinas de interés industrial. Tesis Doctoral, Instituto de Algología Aplicada, Departamento de Biología, Universidad de Las Palmas de Gran Canaria, Las Palmas de Gran Canaria, 130 pp.

Samocha T, J Friker, A Ali, M Shpigel \& A Neori. 2015. Growth and nutrient uptake of the macroalga Gracilaria tikvahiae cultured with the shrimp Litopenaeus vannamei in an Integrated Multi-Trophic Aquaculture (IMTA) system. Aquaculture 446: 263-271.

Schuenhoff A, M Shpigel, I Lupatsch, A Ashkenazi, F Msuya \& A Neori. 2003. A semi-recirculating, integrated system for the culture of fish and seaweed. Aquaculture 221: 167-181.

Shpigel M \& A Neori. 1996. The integrated culture of seaweed, abalone, fish and clams in modular intensive Land-based system: I. proportions of size and projected revenues. Aquacultural Engineering 15: 313-326.

Silva A \& AC Azevedo. 2006. A new version of the statistical assistance software. In: Proceedings of the 4th World Congress on Computers in Agriculture and Natural Resources, pp. 393-396. American Society of Agricultural and Biological Engineers, Orlando.
Soto D \& G Mena. 1999. Filter feeding by the freshwater mussel, Diplodon chilensis, as a biocontrol of salmon farming eutrophication. Aquaculture 171: 65-81.

SUBPESCA. 2003. Política nacional de acuicultura (PNA), 76 pp. Subsecretaria de Pesca, Valparaíso.

Timmons M, J Ebeling, F Wheaton, S Summerfelt \& B Vinci. 2002. Sistemas de recirculación para la acuicultura, 748 pp. Fundación Chile, Santiago.

Troell M, P Rönnbäck, C Halling, N Kautsky \& A Buschmann. 1999. Ecological engineering in aquaculture: use of seaweeds for removing nutrients from intensive mariculture. Journal of Applied Phycology 11: 89-97.

Troell M, C Halling, A Neori, T Chopin, A Buschmann, N Kautsky \& C Yarish. 2003. Integrated mariculture: asking the right questions. Aquaculture 226: 69-90.

Van Khoi L \& R Fotedar. 2011. Integration of western king prawn (Penaeus latisulcatus Kishinouye, 1896) and green seaweed (Ulva lactuca Linnaeus, 1753) in a closed recirculating aquaculture system. Aquaculture 322/323: 201-209.

Vega J, F Álvarez-Gómez, L Güenaga, FL Figueroa \& JL Gómez-Pinchetti. 2020. Antioxidant activity of extracts from marine macroalgae, wild-collected and cultivated, in an integrated multi-trophic aquaculture system. Aquaculture 522: 735088. <https://doi.org/10.1016/j. aquaculture.2020.735088>

Vinatea L. 1999. Aquicultura e desenvolvimento sustentável: subsídios para a formulação de políticas de desenvolvimento da aquicultura brasileira, 310 pp. Editora UFSC, Florianópolis. 